\title{
The Japanese Society of Hypertension—Digest of plan for the future
}

\author{
Koichi Node ${ }^{1} \cdot$ Takuya Kishi $^{2} \cdot$ Atsushi Tanaka $^{1} \cdot$ Hiroshi Itoh $^{3} \cdot$ Hiromi Rakugi $^{4} \cdot$ Yusuke Ohya $^{5} \cdot$ Katsuyuki Miura $^{6} \cdot$ \\ Tomonori Okamura $^{7}$ - Toshihiko Ishimitsu ${ }^{8}$ - Atsuhiro Ichihara ${ }^{9}$ - Masaaki Ito ${ }^{10}$ - Mitsuru Ohishi ${ }^{11}$. \\ Takayoshi Ohkubo $^{12} \cdot$ Hisashi Kai $^{13} \cdot$ Naoki Kashihara $^{14} \cdot$ Kazuomi Kario $^{15} \cdot$ Shigeyuki Saitoh $^{16}$. \\ Takuya Tsuchihashi $^{17}$ - Satoko Nakamura ${ }^{18}$ - Akira Nishiyama $^{19}$ - Naoyuki Hasebe ${ }^{20}$. Jitsuo Higaki ${ }^{21}$. \\ Kouichi Tamura ${ }^{22} \cdot$ Yuhei Kawano ${ }^{23} \cdot$ Takashi Yatabe $^{24}$. \\ Sadayoshi Ito $^{25}$. The JSH Future Plan Working Group and Executive Board Members
}

Received: 5 September 2018 / Accepted: 21 September 2018 / Published online: 11 October 2018

(c) The Japanese Society of Hypertension 2018

Hypertension is the most common cause of cardiovascular diseases and the leading global public health risk for mortality. In our rapidly growing super-aging country, we are facing a time in which advanced innovations in hypertension care are urgently needed. Hence, the Japanese Society of Hypertension (JSH) has newly established a "Future Plan" in an effort to conquer hypertension. In line with this plan, we will explore/perform the best clinical practices for hypertension management and develop positive initiatives for realizing a lively and happy society nationwide.

\section{Slogan}

Good Blood Pressure for Lively 100 Years

$\checkmark$ Goal

Decrease the number of hypertensive individuals by seven million over the next decade, thereby extending the healthy life expectancy.

(An interim appraisal will be discussed at ISH 2022 in Kyoto)

Components

1. Medical system: establishment of a lifetime-care system for individuals with hypertension

- To aim for a lifetime-care society in which all patients can receive high-quality hypertension care according to the guidelines.

- To facilitate the development of a multidisciplinary network that involves the cooperation of specialists, primary care physicians, medical teams, academic societies, administrative agencies, and insurance

Koichi Node

node@cc.saga-u.ac.jp

Extended author information available on the last page of the article providers and to establish a seamless hypertension care system led by hypertension specialists.

2. Academic research: promotion of research in hypertension and embodiment of "Future Medicine"

- To reveal novel underlying mechanisms for the development of more effective therapies that can be distributed worldwide, by using innovative research methods.

- To facilitate "Future Medicine", which enables the prevention, prediction, and control of hypertension, by using artificial intelligence, big-data, and telemedicine via the IoT.

3. Social edification: development of a social model for self-controlled blood pressure

- To work on educational activities for realizing a society in which all people can understand the importance of knowing/controlling their own blood pressure and can adopt healthy lifestyle practices, such as reduction of salt intake, not smoking, and exercise.

- To develop/improve infrastructures suited to regional characteristics and to perform cohesive societybased "Future Medicine" in collaboration with administrative agencies.

\section{Compliance with ethical standards}

Conflict of interest The authors declare that they have no conflict of interest. 


\section{Affiliations}

Koichi Node ${ }^{1} \cdot$ Takuya Kishi $^{2} \cdot$ Atsushi Tanaka $^{1} \cdot$ Hiroshi Itoh $^{3} \cdot$ Hiromi Rakugi $^{4} \cdot$ Yusuke Ohya $^{5} \cdot$ Katsuyuki Miura $^{6} \cdot$ Tomonori Okamura $^{7}$ - Toshihiko Ishimitsu ${ }^{8}$ - Atsuhiro Ichihara ${ }^{9} \cdot$ Masaaki Ito $^{10}$ - Mitsuru Ohishi ${ }^{11}$.

Takayoshi Ohkubo $^{12} \cdot$ Hisashi Kai $^{13} \cdot$ Naoki Kashihara ${ }^{14} \cdot$ Kazuomi Kario $^{15} \cdot$ Shigeyuki Saitoh $^{16}$.

Takuya Tsuchihashi $^{17}$. Satoko Nakamura ${ }^{18}$. Akira Nishiyama ${ }^{19}$. Naoyuki Hasebe ${ }^{20}$. Jitsuo Higaki ${ }^{21}$.

Kouichi Tamura ${ }^{22}$. Yuhei Kawano ${ }^{23}$. Takashi Yatabe ${ }^{24}$.

Sadayoshi Ito $^{25}$. The JSH Future Plan Working Group and Executive Board Members

1 Department of Cardiovascular Medicine, Saga University, Saga, Japan

2 Department of Advanced Risk Stratification for Cardiovascular Diseases, Center for Disruptive Cardiovascular Medicine, Kyushu University, Fukuoka, Japan

3 Department of Endocrinology, Metabolism and Nephrology, School of Medicine, Keio University, Tokyo, Japan

4 Department of Geriatric and General Medicine, Osaka University Graduate School of Medicine, Suita, Japan

5 Department of Cardiovascular Medicine, Nephrology and Neurology, University of the Ryukyus Graduate School of Medicine, Okinawa, Japan

6 Department of Public Health, Shiga University of Medical Science, Otsu, Japan

7 Department of Preventive Medicine and Public Health, Keio University School of Medicine, Tokyo, Japan

8 Department of Cardiology and Nephrology, Dokkyo Medical University Hospital, Mibu, Japan

9 Department of Medicine II, Endocrinology and Hypertension, Tokyo Women's Medical University, Tokyo, Japan

10 Department of Cardiology and Nephrology, Mie University Graduate School of Medicine, Tsu, Japan

11 Department of Cardiovascular Medicine and Hypertension, Graduate School of Medicine and Dental Sciences, Kagoshima University, Kagoshima, Japan

12 Department of Hygiene and Public Health, Teikyo University School of Medicine, Tokyo, Japan

13 Department of Cardiology, Kurume University Medical Center,
Kurume, Japan

14 Department of Nephrology and Hypertension, Kawasaki Medical School, Kurashiki, Japan

15 Division of Cardiovascular Medicine, Department of Medicine, Jichi Medical University School of Medicine, Shimotsuke, Japan

16 School of Health Sciences, Sapporo Medical University, Sapporo, Japan

17 Hypertension Center, Steel Memorial Yawata Hospital, Kitakyushu, Japan

18 Department of Nutritional Science for Well-being, Kansai University of Welfare Sciences, Kashiwara, Japan

19 Department of Pharmacology, Kagawa University Medical School, Kagawa, Japan

20 Division of Cardiology, Nephrology, Pulmonology and Neurology, Department of Internal Medicine, Asahikawa Medical University, Asahikawa, Japan

21 Minami-Matsuyama Hospital, Ehime University, Matsuyama, Japan

22 Department of Medical Science and Cardio-Renal Medicine, Yokohama City University Graduate School of Medicine, Yokohama, Japan

23 Department of Medical Technology, Teikyo University Fukuoka, Omuta, Japan

24 Yatabe \& Momii Law Office, Tokyo, Japan

25 Division of Nephrology, Endocrinology and Hypertension, Tohoku University Graduate School of Medicine, Sendai, Japan 